\title{
Biceps-Related Physical Findings Are Useful to Prevent Misdiagnosis of Cervical Spondylotic Amyotrophy as a Rotator Cuff Tear
}

\author{
Eiichiro Iwata ${ }^{1}$, Hideki Shigematsu $^{1}$, Kazuya Inoue ${ }^{1}$, Takuya Egawa ${ }^{1}$, Masato Tanaka ${ }^{1}$, \\ Akinori Okuda ${ }^{2}$, Yasuhiko Morimoto ${ }^{1}$, Keisuke Masuda ${ }^{1}$, Yusuke Yamamoto ${ }^{1}$, \\ Yoshihiro Sakamoto ${ }^{3}$, Munehisa Koizumi ${ }^{4}$, Yasuhito Tanaka ${ }^{1}$ \\ ${ }^{1}$ Department of Orthopaedic Surgery, Nara Medical University, Nara, Japan \\ ${ }^{2}$ Department of Emergency and Critical Care Medicine, Nara Medical University, Nara, Japan \\ ${ }^{3}$ Department of Orthopaedic Surgery, Saiseikai Gose Hospital, Nara, Japan \\ ${ }^{4}$ Department of Spine Surgery, Nara Prefecture General Medical Center, Nara, Japan
}

\section{Study Design: Case-control study.}

Purpose: The aim of the present study was to identify physical findings useful for differentiating between cervical spondylotic amyotrophy (CSA) and rotator cuff tears to prevent the misdiagnosis of CSA as a rotator cuff tear.

Overview of Literature: CSA and rotator cuff tears are often confused among patients presenting with difficulty in shoulder elevation.

Methods: Twenty-five patients with CSA and 27 with rotator cuff tears were enrolled. We included five physical findings specific to CSA that were observed in both CSA and rotator cuff tear patients. The findings were as follows: (1) weakness of the deltoid muscle, (2) weakness of the biceps muscle, (3) atrophy of the deltoid muscle, (4) atrophy of the biceps muscle, and (5) swallow-tail sign (assessment of the posterior fibers of the deltoid).

Results: Among 25 CSA patients, 10 (40.0\%) were misdiagnosed with a rotator cuff tear on initial diagnosis. The sensitivity and specificity of each physical finding were as follows: (1) deltoid weakness (sensitivity, 92.0\%; specificity, 55.6\%), (2) biceps weakness (sensitivity, 80.0\%; specificity, 100\%), (3) deltoid atrophy (sensitivity, 96.0\%; specificity, 77.8\%), (4) biceps atrophy (sensitivity, 88.8\%; specificity, 92.6\%), and (5) swallow-tail sign (sensitivity, 56.0\%; specificity, 74.1\%). There were statistically significant differences in each physical finding.

Conclusions: CSA is likely to be misdiagnosed as a rotator cuff tear; however, weakness and atrophy of the biceps are useful findings for differentiating between CSA and rotator cuff tears to prevent misdiagnosis.

Keywords: Biceps muscle; Deltoid muscle; Rotator cuff; Cervical spondylosis; Differential diagnosis

Received Apr 25, 2017; Revised May 15, 2017; Accepted Jun 7, 2017

Corresponding author: Eiichiro Iwata

Department of Orthopaedic Surgery, Nara Medical University, 840 Shijo-cho, Kashiharashi, Nara 634-8522, Japan

Tel: +81-744-29-8873, Fax: +81-744-29-4902, E-mail: iwata@naramed-u.ac.jp 


\section{Introduction}

The causes of a difficulty in shoulder elevation are often difficult to identify because this complaint might originate from shoulder and cervical spine root disorders. In addition, the age at occurrence of cervical spine and shoulder pathologies is very similar, particularly among the aging population $[1,2]$. Therefore, cervical spine and shoulder pathologies are often misdiagnosed. Among these conditions, cervical spondylotic amyotrophy (CSA) and rotator cuff tears are often confused because the main symptom in both is difficulty in shoulder elevation.

CSA is classified into proximal and distal types [3-5]. The clinical characteristics of the proximal type of CSA are muscle atrophy in the upper extremities, no or insignificant sensory deficit and lower extremity symptoms, and weakness of the deltoid and biceps, which often causes drop shoulder [3-7]. Patients with CSA are frequently referred to orthopedic surgeons specializing in shoulder surgery.

Usually, conservative therapy is effective for CSA, but surgery is occasionally needed if conservative therapy is ineffective. The duration of symptoms is a risk factor for poor outcome after surgical treatment $[8,9]$; hence, early diagnosis is very important. The aim of the present study was to identify physical findings useful for differentiating between CSA and rotator cuff tears to prevent the misdiagnosis of CSA as a rotator cuff tear.

\section{Materials and Methods}

\section{Patients}

After receiving approval from the Institutional Review Board of Nara Medical University, the present study was performed (IRB approval no., 1135). Between January 2014 and August 2015, consecutive patients with CSA who presented to our clinic (CSA group) and those with rotator cuff tears who underwent surgery at our hospital (rotator cuff tear group) were enrolled.

\section{Diagnosis of cervical spondylotic amyotrophy}

The proximal type of CSA was diagnosed by an orthopedic surgeon specializing in spine surgeries according to the following criteria: (1) the chief complaint was difficulty in shoulder elevation with no or insignificant sensory deficit and no lower extremity symptoms; (2) magnetic resonance imaging (MRI) or computed tomography (CT) myelography revealed C5 or C6 nerve or anterior horn compression; and (3) electromyography was performed by a neurologist, which enabled the exclusion of other diseases such as amyotrophic lateral sclerosis and motor neuron disease.

\section{Diagnosis of rotator cuff tears}

Rotator cuff tear was diagnosed by an orthopedic surgeon specializing in shoulder surgeries according to the following criteria: (1) MRI revealed a tear and (2) the surgeon who operated recognized the tear intraoperatively. We excluded patients with cuff tear arthropathy.

\section{Physical findings specific to cervical spondylotic amyotrophy}

We selected five physical findings specific to CSA [3$7,10,11$ ] (originating from disorders of the C5 or C6 nerve or anterior horn). The findings were as follows: (1) weakness of the deltoid muscle, (2) weakness of the biceps muscle, (3) atrophy of the deltoid muscle, (4) atrophy of the biceps muscle, and (5) swallow-tail sign (assessment of the posterior fibers of the deltoid) [10]. These physical findings were assessed in both CSA and rotator cuff tear patients. Muscle strength was determined on the basis of manual muscle testing (MMT) [12]. We defined muscle weakness as an MMT of $\leq 3$ or an MMT of 4 with the strength of the affected side being less than that of the intact contralateral side, considering that it is often difficult to distinguish between an MMT of 4 and 5 because of considerable dependence on the judgment of the testers. Evaluations of muscle atrophy were based on laterality. We assessed these five physical findings in the CSA patients immediately upon the diagnosis of CSA and in the rotator cuff tear patients immediately before surgery.

\section{Evaluation of the misdiagnosis rate and the sensitiv- ity and specificity of the physical findings}

We calculated the rate of CSA patients misdiagnosed as having a rotator cuff tear on initial diagnosis at other clinics or hospitals and referred to an orthopedic surgeon specializing in shoulder surgery at our hospital. We calculated the sensitivity and specificity of each physical 
Table 1. Demographic data

\begin{tabular}{lccc} 
Characteristic & Cervical spondylotic amyotrophy group & Rotator cuff tear group & $p$-value \\
No. of patient & 25 & 27 & - \\
Sex & 17 & 17 & 0.703 \\
\hline Male & 8 & 10 & $0.022^{*}$ \\
\hline Female & $73 \pm 9.0$ & $68 \pm 8.3$ & 0.3 \\
\hline Age (yr) &
\end{tabular}

Values are presented as number or mean \pm standard deviation.

$p<0.05$ (statistically significant).

examination, and statistical analysis was performed using a chi-square test or Fisher's exact probability test. In other analyses, the differences in quantitative characteristics such as age were determined using Student $t$-test. Differences in qualitative characteristics such as sex were analyzed using the chi-square test. All statistical analyses were performed using IBM SPSS ver. 23.0 for Windows (IBM Corp., Armonk, NY, USA). A $p$-value of $<0.05$ was considered statistically significant.

\section{Results}

\section{Demographics}

Twenty-five patients with CSA and 27 with rotator cuff tears were enrolled in the present study. The CSA group comprised 17 men and eight women; the rotator cuff tear group comprised 17 men and 10 women. The mean age was 69.8 and 64.1 years in the CSA and rotator cuff tear groups, respectively. There were no significant differences in the sexes of patients between the two groups; however, the mean age was significantly higher in the CSA group (Table 1).

\section{Site of rotator cuff tears}

The sites of rotator cuff tears are presented in Table 2. The supraspinatus tendon was involved in all 27 cases.

\section{Misdiagnosis rate}

Among 25 CSA patients, 10 (40.0\%) were misdiagnosed as having a rotator cuff tear on initial diagnosis by orthopedic clinics before being referred to our department.
Table 2. Site of rotator cuff tears

\begin{tabular}{lc} 
Torn tendons & No. of patient \\
SSP & 15 \\
SSP+ISP & 5 \\
SSP+SS & 3 \\
SSP+ISP+SS & 4 \\
Total no. & 27 \\
\hline
\end{tabular}

SSP, supraspinatus; ISP, infraspinatus; SS, subscapularis.

\section{Sensitivity and specificity of physical examination}

The sensitivity and specificity of each physical examination were as follows: (1) weakness of the deltoid muscle (sensitivity, 92.0\%; specificity, 55.6\%), (2) weakness of the biceps muscle (sensitivity, $80.0 \%$; specificity 100\%), (3) atrophy of the deltoid muscle (sensitivity, $96.0 \%$; specificity, 77.8\%), (4) atrophy of the biceps muscle (sensitivity, $88.8 \%$; specificity, 92.6\%), and (5) swallow-tail sign (sensitivity, $56.0 \%$; specificity, $74.1 \%$ ). There were statistically significant differences in each of the physical findings (Table 3).

\section{Discussion}

In the present study, 10 patients (40.0\%) with CSA were misdiagnosed as having rotator cuff tears on initial diagnosis. Therefore, we surmise that CSA is highly likely to be misdiagnosed as a rotator cuff tear. Some prior reports discuss the differential diagnosis between cervical and shoulder pathologies with respect to the causes of localized shoulder pain $[1,2]$. However, to the best of our knowledge, there are no reports on the differential diagnosis between cervical and shoulder pathologies with respect to the causes of difficulty in shoulder elevation.

CSA is classified into two types based on its clinical features. Proximal-type CSA presents with atrophy and 
Table 3. Sensitivity and specificity of each physical finding

\begin{tabular}{|c|c|c|c|}
\hline Variable & Sensitivity (\%) & Specificity (\%) & $p$-value \\
\hline Weakness of the deltoid muscle & 92.0 & 55.6 & $<0.001^{*}$ \\
\hline Weakness of the biceps muscle & 80.0 & 100.0 & $<0.001^{*}$ \\
\hline Atrophy of the deltoid muscle & 96.0 & 77.8 & $<0.001^{*}$ \\
\hline Atrophy of the biceps muscle & 88.8 & 92.6 & $<0.001^{*}$ \\
\hline Swallow-tail sign & 56.0 & 74.1 & $0.027^{*}$ \\
\hline
\end{tabular}

" $p<0.05$ (statistically significant).

weakness of the deltoid and biceps and originates from the C5 or C6 roots or anterior horns [3-7]. Distal-type CSA presents with atrophy and the weakness of extensor and flexor muscles of the fingers and intrinsic hand muscles and originates from the $\mathrm{C} 7-\mathrm{T} 1$ roots or anterior horns [3-7]. The major symptom of proximal-type CSA is difficulty in shoulder elevation caused by weakness of the deltoid and biceps. In addition, the age at which cervical spine and shoulder pathologies occur is very similar, particularly among the aging population $[1,2]$. Therefore, proximal-type CSA is often misdiagnosed as a shoulder disorder, particularly as a rotator cuff tear. Conservative therapy is usually effective in CSA; however, if it is ineffective, surgery is needed $[8,9]$. Tauchi et al. [8] reported that early surgery was recommended for CSA patients in whom conservative treatment was unsuccessful based on the clinical status before surgery, including symptom duration. Uchida et al. [9] reported that a long preoperative period was strongly correlated with low muscle power improvement. Early diagnosis is therefore vital.

The diagnosis of CSA is comprehensively based on physical findings, disease course, findings of imaging modalities such as MRI and CT myelography, and electromyographic findings [11,13-15]. However, imaging studies and electromyography generally cause a delay in the diagnosis and entail a high cost, whereas physical findings are convenient to assess and enable rapid diagnosis [1]. Therefore, we identified the physical findings useful for differentiating between CSA and rotator cuff tears to prevent the misdiagnosis of CSA as a rotator cuff tear, particularly on initial diagnosis.

We chose five physical findings specific to CSA and observed them in both CSA and rotator cuff tear patients. Nishijima et al. [10] reported that to fully extend the shoulders, similar to a swallow tail, the posterior fibers of the deltoid are essential. They named this physical finding the swallow-tail sign; this finding enables the evaluation of the posterior fibers of the deltoid [10]. Each finding involved in disorders of the $\mathrm{C} 5$ or $\mathrm{C} 6$ nerve roots or anterior horn was specific to CSA. We calculated the sensitivity and specificity of each physical finding.

Physical findings related to the deltoid, such as weakness and atrophy of deltoid and the swallow-tail sign, were highly sensitive but not highly specific. Therefore, these physical findings might be positive, to some extent, in patients with rotator cuff tears. Kido et al. [16] reported that the average decrease in the muscle volume of shoulders with rotator cuff tears is $19 \%-30 \%$ in the rotator cuff muscles and $29 \%$ in the deltoid muscle. Therefore, deltoid function might be affected by a rotator cuff tear.

The physical findings related to the biceps, such as weakness and atrophy of the biceps, were both highly sensitive and specific. Therefore, these physical findings could be useful for differentiating between CSA and rotator cuff tears.

Our study has several limitations. First, we did not evaluate the coexistence of CSA and a rotator cuff tear, i.e., patients with CSA, except those misdiagnosed with rotator cuff tears on initial diagnosis, did not undergo examinations for rotator cuff tears, and patients with rotator cuff tears did not undergo examinations for CSA. Second, we did not evaluate inter- and intra-observer errors. However, the evaluation of muscle weakness was based on MMT, which is generally accepted worldwide as a reliable test for assessing muscle strength [12]. Evaluations of muscle atrophy and the swallow-tail sign were based on laterality; therefore, inter- and intra-observer errors might have been fewer. Third, we did not conduct a power analysis to determine the appropriate sample size before conducting the present study; the number of cases is also comparatively few. However, because there was a significant difference in each physical finding, we must consider the possibility that a type 2 error (caused by a small sample size) might not have occurred. 
We believe that physical findings related to the biceps muscle play a vital role in the initial differential diagnosis between CSA and rotator cuff tears. Imaging methods such as MRI, CT myelography, and electromyography are needed for a more accurate diagnosis. We suggest that in patients with suspected disorders of shoulder elevation, physicians should perform a physical examination to evaluate the biceps, including tests for weakness and atrophy. Thus, we can prevent the misdiagnosis of CSA as a rotator cuff tear and a delay in diagnosing CSA. If these physical findings are positive, more accurate diagnostic tools such as MRI, CT, and electromyography can be used.

\section{Conclusions}

CSA is highly likely to be misdiagnosed as a rotator cuff tear. Weakness and atrophy of the biceps are useful findings to differentiate between CSA and rotator cuff tears and prevent misdiagnosis.

\section{Conflict of Interest}

No potential conflict of interest relevant to this article was reported.

\section{References}

1. Gumina S, Carbone S, Albino P, Gurzi M, Postacchini F. Arm Squeeze Test: a new clinical test to distinguish neck from shoulder pain. Eur Spine J 2013;22:155863.

2. Katz JS, Saperstein DS, Wolfe G, et al. Cervicobrachial involvement in diabetic radiculoplexopathy. Muscle Nerve 2001;24:794-8.

3. Kameyama T, Ando T, Yanagi T, Yasui K, Sobue G. Cervical spondylotic amyotrophy: magnetic resonance imaging demonstration of intrinsic cord pathology. Spine (Phila Pa 1976) 1998;23:448-52.

4. Keegan JJ. The cause of dissociated motor loss in the upper extremity with cervical spondylosis. J Neurosurg 1965;23:528-36.

5. Matsunaga S, Sakou T, Imamura T, Morimoto N. Dissociated motor loss in the upper extremities: clinical features and pathophysiology. Spine (Phila Pa 1976) 1993;18:1964-7.

6. Brain WR, Northfield D, Wilkinson M. The neurological manifestations of cervical spondylosis. Brain 1952;75:187-225.

7. Sobue I, Kato H, Yanagi T. Clinical characteristics and classification of cervical spondylotic myelopathy. Rinsho Seikeigaku 1975;10:999-1006.

8. Tauchi R, Imagama S, Inoh H, et al. Risk factors for a poor outcome following surgical treatment of cervical spondylotic amyotrophy: a multicenter study. Eur Spine J 2013;22:156-61.

9. Uchida K, Nakajima H, Yayama T, et al. Anterior and posterior decompressive surgery for progressive amyotrophy associated with cervical spondylosis: a retrospective study of 51 patients. J Neurosurg Spine 2009;11:330-7.

10. Nishijma N, Yamamuro T, Fujio K, Ohba M. The swallow-tail sign: a test of deltoid function. J Bone Joint Surg Br 1995;77:152-3.

11. Jin X, Jiang JY, Lu FZ, Xia XL, Wang LX, Zheng CJ. Electrophysiological differences between Hirayama disease, amyotrophic lateral sclerosis and cervical spondylotic amyotrophy. BMC Musculoskelet Disord 2014;15:349.

12. Daniels L, Worthingham C. Muscle testing: techniques of manual examination. 4th ed. Philadelphia: WB Saunders; 1980.

13. Imajo Y, Kato Y, Kanchiku T, Suzuki H, Taguchi T. Pathology and prognosis of proximal-type cervical spondylotic amyotrophy: new assessment using compound muscle action potentials of deltoid and biceps brachii muscles. Spine (Phila Pa 1976) 2011;36:E47681.

14. Jiang SD, Jiang LS, Dai LY. Cervical spondylotic amyotrophy. Eur Spine J 2011;20:351-7.

15. Shibuya R, Yonenobu K, Yamamoto K, et al. Acute arm paresis with cervical spondylosis: three case reports. Surg Neurol 2005;63:220-8.

16. Kido T, Itoi E, Konno N. Atrophy of the deltoid and rotator cuff muscles in shoulders with rotator cuff tears. Tohoku Arch Orthop Surg Traumatol 1997;41:109-11. 